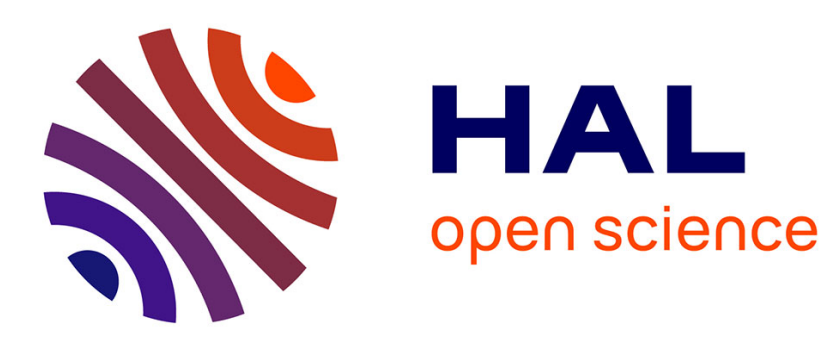

\title{
Finite-Element Simulation of the Depolarization Factor of Arbitrarily Shaped Inclusions
}

\author{
Abdelilah Mejdoubi, Christian Brosseau
}

\section{To cite this version:}

Abdelilah Mejdoubi, Christian Brosseau. Finite-Element Simulation of the Depolarization Factor of Arbitrarily Shaped Inclusions. 2013, pp.4. hal-00918337

\section{HAL Id: hal-00918337 \\ https://hal.science/hal-00918337}

Submitted on 31 Dec 2013

HAL is a multi-disciplinary open access archive for the deposit and dissemination of scientific research documents, whether they are published or not. The documents may come from teaching and research institutions in France or abroad, or from public or private research centers.
L'archive ouverte pluridisciplinaire HAL, est destinée au dépôt et à la diffusion de documents scientifiques de niveau recherche, publiés ou non, émanant des établissements d'enseignement et de recherche français ou étrangers, des laboratoires publics ou privés. 


\title{
Finite-Element Simulation of the Depolarization Factor of Arbitrarily Shaped Inclusions
}

\author{
A. Mejdoubi ${ }^{1}$ and C. Brosseau ${ }^{1,2}$ \\ ${ }^{1}$ Laboratoire d'Electronique et Systèmes de Télécommunications, Université de Bretagne Occidentale, CS 93837 Brest Cedex 3, \\ France \\ ${ }^{2}$ Département de Physique, Université de Bretagne Occidentale, 29238 Brest Cedex 3, France
}

\begin{abstract}
An understanding of the polarization characteristics is vital to the rational design of future dielectric nanostructures. In this work, a finite-element methodology has been applied to simulate 2-D two-phase heterostructures containing a dielectric inclusion with arbitrary shape. The development does not impose any restriction on the shape of the inclusion. Given the paucity of experimental and numerical data, we set out to investigate systematically the trends that shape and permittivity contrast between the inclusion and the host matrix have on the depolarization factor (DF). The effect of the first- versus second-order concentration virial coefficient on the value of the DF is considered for a variety of inclusion shapes and a large set of material properties. Our findings suggest that the DF for a 2-D inclusion is highly tunable depending on the choice of these parameters. These results can provide a useful insight for the design of artificial two-phase heterostructures with specific polarization properties.
\end{abstract}

Index Terms-Composite materials, depolarization factor, effective permittivity, finite element.

\section{INTRODUCTION}

$\mathbf{T}$ HE MAIN goal of this paper is the depolarization factor (DF) of arbitrarily shaped polarizable inclusion in 2-D heterostructures. Since analytical calculation of the response of an arbitrary composite material is typically intractable, numerical simulation is the standard method to extract the effective properties of these complex media. For that purpose we use a finite-element (FE) methodology to derive the effective permittivity of the mixed medium. As an illustrative application of this technique, we consider a number of 2-D systems with different kinds of inclusion geometry and orientation with respect to the applied electric field. One aim of the calculations outlined below is to illustrate how the DF depends on the permittivity ratio between the inclusion and the host matrix. We devote a significant amount of our efforts to demonstrate that the DF is strongly influenced by the boundaries roughness.

\section{Methodology And Computational AsPects}

\section{A. Depolarization Factor}

The dielectric 2-D heterostructure is divided into two phases: one phase characterized by different shapes, isotropic permittivity $\varepsilon_{2}$, and surface fraction $\phi_{2}$ distributed in another phase characterized by isotropic permittivity $\varepsilon_{1}$ and surface fraction $\phi_{1}$. Anisotropy can arise out of some asymmetry in the microstructure, e.g., distribution of oriented nondiscoidal inclusions in a matrix. In this case, the DF is the tensor

$$
\overleftrightarrow{A}=\left[\begin{array}{ll}
A_{\mathrm{xx}} & A_{\mathrm{xy}} \\
A_{\mathrm{yx}} & A_{\mathrm{yy}}
\end{array}\right]
$$

Digital Object Identifier 10.1109/TMAG.2007.916492 in a Cartesian system of coordinates. The trace of $\overleftrightarrow{A}$ is unity, and therefore, $0 \leqslant A_{\mathrm{ij}} \leqslant 1, i=x, y$. For other isotropic cases, the DF is a scalar $\stackrel{\leftrightarrow}{A}=A \overleftrightarrow{1}$. For simplicity, the following formulation is written in terms of scalar quantities. However, the procedure is easily generalized by considering permittivity and depolarization tensors.

In many instances the effective permittivity $\varepsilon$ can be scaled to collapse to a common set of master curves described by

$$
\frac{\varepsilon}{\varepsilon_{1}}=f\left(\frac{\varepsilon_{2}}{\varepsilon_{1}}, \phi_{2}, A\right)
$$

where $A(0 \leqslant A \leqslant 1)$ is a functional of inclusion shape and permittivity mismatch only. By now, a broad choice of analytic expressions is available for the function $f$ which may score very well if compared to experimental or numerical data [1], [2]. For example, the Maxwell Garnett (MG) [1], [2] form for $f$ is given by

$$
f\left(\frac{\varepsilon_{2}}{\varepsilon_{1}}, \phi_{2}, A\right)=1+\frac{\phi_{2}\left(\frac{\varepsilon_{2}}{\varepsilon_{1}}-1\right)}{1+A\left(1-\phi_{2}\right)\left(\frac{\varepsilon_{2}}{\varepsilon_{1}}-1\right)} .
$$

Note that the roles of host and inclusion in (2) are not reciprocal. In other instances, the function $f$ appearing in the right-hand side of (1) is more complex, e.g., for Böttcher equation [also termed symmetric Bruggeman (SBG)] [1], [2], it can be computed as in (3), shown at the bottom of the next page.

Note that the roles of host and inclusion media are reciprocal. In practice, laboratory or numerical data can be approximated arbitrarily accurately with (2) or (3) only in the dilute limit, i.e., when $\phi_{2}$ is sufficiently small.

The question now is whether the MG and SBG analyses give different set of $A$ values. At the heart of this study $A$ enters a linearized version of (1) as fitting parameter. For the dilute limit we expanded (2) and (3) to first-order in $\phi_{2}$. In its simples form this series expansion is 


$$
f\left(\frac{\varepsilon_{2}}{\varepsilon_{1}}, \phi_{2}, A\right)=1+\alpha \phi_{2}+O\left(\phi_{2}^{2}\right)
$$

where

$$
\alpha_{\mathrm{MG}}=\alpha_{\mathrm{SBG}}=\alpha=\frac{1}{A+\frac{1}{\frac{\varepsilon_{2}}{\varepsilon_{1}}-1}} .
$$

This expression leads to three results. First, we show that the leading first-order corrections are similar for MG and SBG equations. Thus, the value of $A$ we obtain is, up to the level of approximation based on the dilute limit, model-independent. Second, (4) allows the determination of $A$ which is independent of $\phi_{2}$.

Before examining the trends of the DF, it is instructive to look at the series expansion to second-order in $\phi_{2}$. The second-order expansion of $f$ may be written as

$$
f\left(\frac{\varepsilon_{2}}{\varepsilon_{1}}, \phi_{2}, A\right)=1+\tilde{\alpha} \phi_{2}+\tilde{\beta} \phi_{2}^{2}+\mathrm{O}\left(\phi_{2}^{3}\right)
$$

with coefficients

$$
\begin{aligned}
\tilde{\beta}_{\mathrm{MG}} & =\frac{1}{A+\frac{2}{\frac{\varepsilon_{2}}{\varepsilon_{1}}-1}+\frac{1}{A}\left(\frac{1}{\frac{\varepsilon_{2}}{\varepsilon_{1}}-1}\right)^{2}} \\
\tilde{\beta}_{\mathrm{SBG}} & =\frac{\frac{\varepsilon_{2}}{\varepsilon_{1}}}{A^{2}\left(\frac{\varepsilon_{2}}{\varepsilon_{1}}-1\right)\left(1+\frac{1}{A\left(\frac{\varepsilon_{2}}{\varepsilon_{1}}-1\right)}\right)^{3}}
\end{aligned}
$$

obtained using the computer package Maple. Thus, at higher than linear order approximation, the $A$ values are model-dependent and can differ from those determined by using the firstorder approximation depending on the range of $\phi_{2}$ which is considered. In general, this comparison depends on the system under consideration and the level of accuracy required. Such effect will be discussed in this work. For the purposes of the present discussion, the DF will be analyzed by fitting the effective permittivity data by means of (4) for $\phi_{2}<\phi_{2 c o} \approx 0.05$, or using (5) for $\phi_{2}<\phi_{2 \text { co }} \approx 0.10$.

\section{B. Finite-Element Methodology}

We consider a general 2-D system which can be defined as a bounded domain in the complex plane of surface $\Omega$ which has effective permittivity $\varepsilon$, in which there is no source charge, as illustrated in Fig. 1. Solving the problem at hand means finding expressions for the scalar potential $V$ and electric field $E=$ $-\nabla V$ everywhere within the domain $\Omega$. The local potential distribution inside $\Omega$ is given by the conservation of electric displacement flux through the "surface" $S$, i.e., Laplace partial differential equation

$$
\nabla \cdot(\varepsilon(\boldsymbol{r}) \nabla V)=0
$$

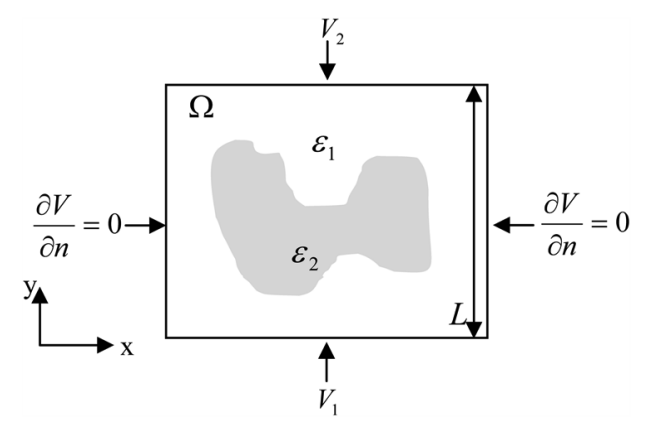

Fig. 1. Cartoon sketch of the unit square cell of a typical 2-D composite structure containing a single inclusion (shaded region). The model space can simulate a capacitor by applying a potential difference between the top and bottom faces of the model space. The evaluation of the effective permittivity, along the direction corresponding to the applied field, i.e., $\varepsilon=\varepsilon_{y}$, requires that the conservation of the electric displacement flux through the "surface" $S$ has to be solved subject to appropriate the relevant boundary conditions for the potential. We fix $V_{1}=0 \mathrm{~V}$ and $V_{2}=1 \mathrm{~V}$ and assume that $(\partial \mathrm{V} / \partial n)=0$ on the other side faces. $L$ and $S$ have both been set to unity.

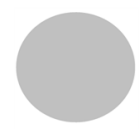

(a)

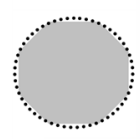

(f)

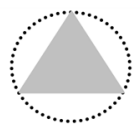

(b)

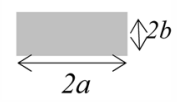

(g)

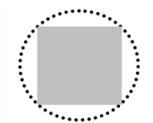

(c)

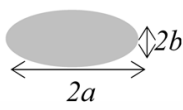

(h)

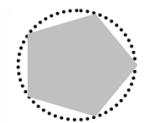

(d)

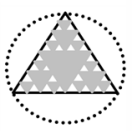

(i)

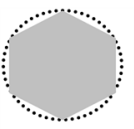

(e)

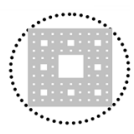

(j)
Fig. 2. Schematic diagrams of the structural motifs of the inhomogeneous mixtures considered in this work. (a) Disk. (b) Equilateral triangle. (c) Square. (d) Regular pentagon. (e) Regular hexagon. (f) Regular octogon. (g) Rectangle of dimensions $2 a$ and $2 b$. (h) Ellipse of semimajor axis $a$ and semiminor axis $b$. (i) Sierpinski triangle (third iteration). (j) Sierpinski square (third iteration).

where $\varepsilon(\boldsymbol{r})$ and $V$ are the local permittivity and potential, respectively. In the case at hand, the effective permittivity along the direction corresponding to the applied field, i.e., $\varepsilon=\varepsilon_{y}$, is found by integration via $\int_{S} \varepsilon_{1}(\partial V / \partial n)_{1}=\varepsilon\left(V_{2}-V_{1 / L}\right) S$, where $V_{2}-V_{1}$ denotes the difference of potential imposed in the $y$-direction, $L$ is the composite thickness in the same direction, and $S$ is the "surface" of the unit cell perpendicular to the applied field. The potential on the top face of the square, $V_{2}$, is fixed at a value of $1 \mathrm{~V}$, while that on the bottom face, $V_{1}$, is fixed at $0 \mathrm{~V}$. One then solves (6) subject to appropriate boundary conditions. The algorithm was similar to that used in [3]. For simplicity, we will focus our discussion on 2-D deterministic two-phase heterostructures. In all cases, the simulation cell $\Omega$ is a square of length $L=1$.

Periodic boundary conditions are enforced in the $x$ direction for these structures. All data were obtained using the FE element as implemented in the commercial finite-element solver Comsol

$$
f\left(\frac{\varepsilon_{2}}{\varepsilon_{1}}, \phi_{2}, A\right)=\frac{1-A\left(1+\frac{\varepsilon_{2}}{\varepsilon_{1}}\right)+\phi_{2}\left(\frac{\varepsilon_{2}}{\varepsilon_{1}}-1\right) \pm \sqrt{\left[1-A\left(1+\frac{\varepsilon_{2}}{\varepsilon_{1}}\right)+\phi_{2}\left(\frac{\varepsilon_{2}}{\varepsilon_{1}}-1\right)\right]^{2}+4 A(1-A) \frac{\varepsilon_{2}}{\varepsilon_{1}}}}{2(1-A)}
$$




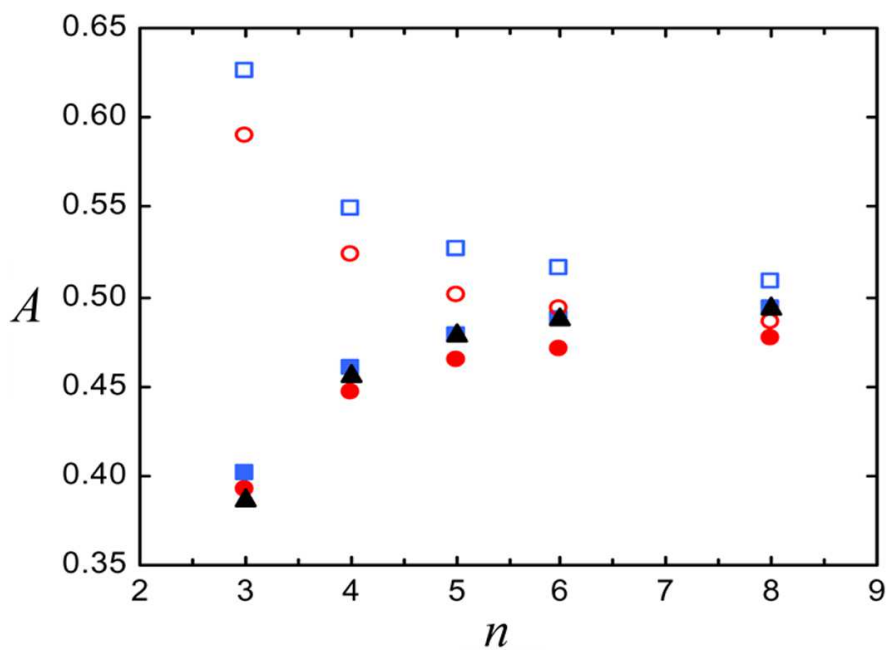

Fig. 3. Number of vertices dependence of DF for polygons. Open circles and squares correspond to the values of $A$ deduced from first-order and second-order approximation, respectively, for $\left(\varepsilon_{2} / \varepsilon_{1}\right)=(20 / 2)$, whereas solid circles and squares the values of $A$ deduced from first-order and second-order approximation, respectively, for $\left(\varepsilon_{2} / \varepsilon_{1}\right)=(1 / 100)$. For comparison, the DF data (solid triangles) of Garboczi and Douglas [4] are also shown for polygons in the limit $\left(\varepsilon_{2} / \varepsilon_{1}\right) \rightarrow \infty$.

Multiphysics and the procedure sorted out $\varepsilon$ on a personal computer (PC) with a Pentium IV processor $(3 \mathrm{GHz})$. Comsol Multiphysics permits the closely controlled generation of FE meshes through the use of input files containing complete instructions for node-by-node and element-by-element mesh specification, along with imposition of boundary conditions. In this work, the $y$-axis was defined as pointing in the direction of the applied electric field.

Before proceeding, it is useful first to present in Fig. 2 the relatively simple prototypical types of inclusion that were selected in this study: regular $n$-gons, ellipse, rectangle, Sierpinski square, and triangle fractal structures. The ellipse is characterized with semimajor axis $a$ and semiminor axis $b$. The dimensions of the rectangle are $2 a \times 2 b$.

As noted in the Introduction, despite concentrated efforts, very few exact results on the DF are known. The only data to which to compare our calculated DF are the values given by Douglas and Garboczi [4], [6] for the $n$-gons inclusion which are in good agreement with our value (see below).

\section{RESULTS AND DISCUSSION}

Unless otherwise noted the DF values plotted in the figures are those obtained using (5).

\section{A. Effect of Varying the Inclusion Shape and Orientation With Respect to the Applied Electric Field}

Consider first the case of polygons (Fig. 3). Remarkably, we found a general trend in series of polygons, i.e., the values of $A$ are substantially higher as the number of vertices of the inclusion increases when respectivelyl $\left(\varepsilon_{2} / \varepsilon_{1}\right)>1$. Overall, this trend appears to be independent of permittivity ratio over the range of permittivity ratios considered. For polygons, $A$ achieves its absolute maximum for disk when respectively

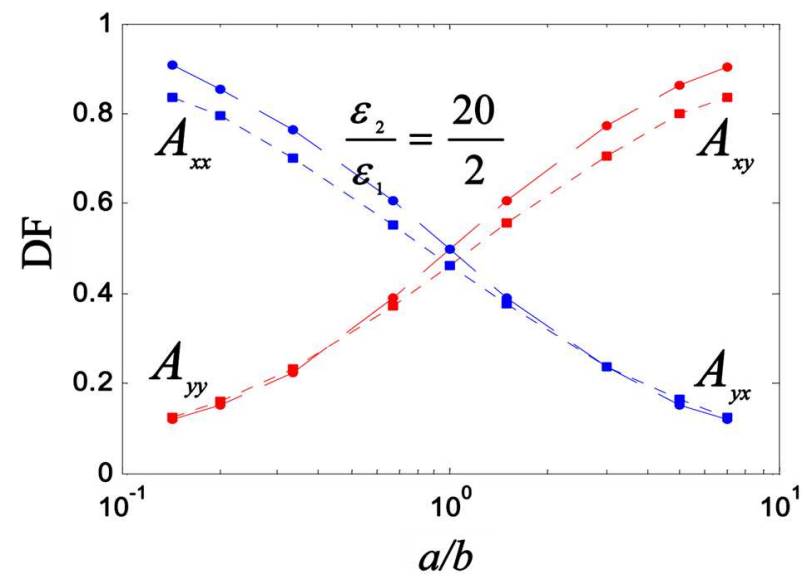

Fig. 4. Aspect ratio dependence of DF tensor components for ellipse ( $\bullet$ ) and rectangular $(\mathbf{\square})$ inclusion displayed on a semilog plot. $\left(\varepsilon_{2} / \varepsilon_{1}\right)=(20 / 2)$. The lines are guides for the eyes.

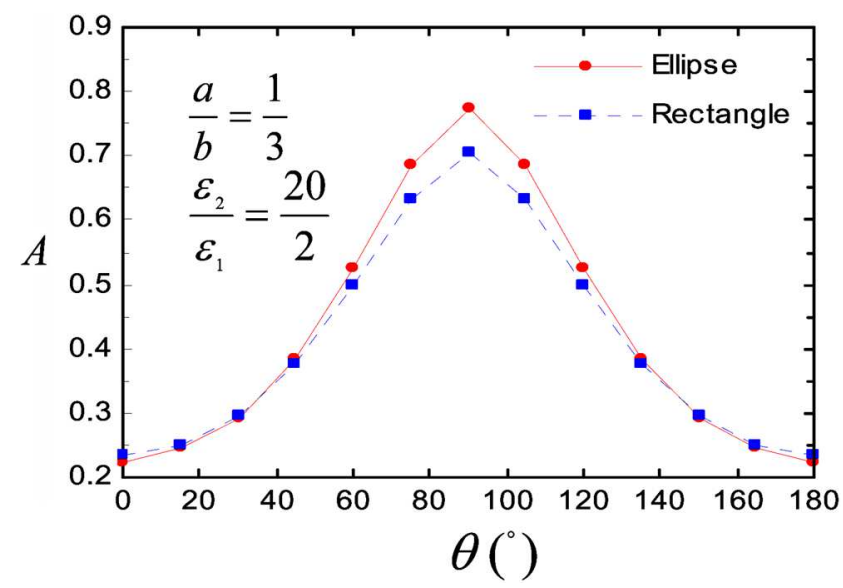

Fig. 5. Plot of the angular dependence of the DF. Inclusion rotations are performed about the $y$-axis. Symbols are: $(\bullet)$ ellipse and $(\boldsymbol{\square})$ rectangle. The permittivity contrast is set to $\left(\varepsilon_{2} / \varepsilon_{1}\right)=(20 / 2)$, and the aspect ratio is $(a / b)=$ $(1 / 3)$. The lines are guides for the eyes.

$\left(\varepsilon_{2} / \varepsilon_{1}\right)>1$, i.e., $A \rightarrow(1 / 2)$ in the limit $n \rightarrow \infty$. A comparison of the two approximations, i.e., (4) and (5), indicates that while there are slight differences in the actual values, the resulting DF are comparable. It is difficult to decide which of these procedures provides the better description in general. The data from Douglas and Garboczi [4], [6] are presented for comparison. They are close to the current results.

The effect of anisotropic inclusions, i.e., ellipse and rectangle, for an electric field polarized in the $x$ - and $y$-directions is now considered. As displayed in Fig. 4, there is significant effect of inclusion asymmetry on DF. The identical mild " $\mathrm{S}$ "-shaped profile is observed for both inclusions.

For purpose of comparison, we plot the DF tensor components for two polarizations in Fig. 4. From this graph, one directly verifies that $\operatorname{tr}(\overleftrightarrow{A})=1$. Our simulation indicates that a difference of about one order of magnitude can be evidenced between the longitudinal and transverse DFs. But even more remarkable is the effect of the orientation of ellipse and rectangle with respect to the applied electric field, qualitatively almost identical for both inclusions. This is illustrated in Fig. 5, where 


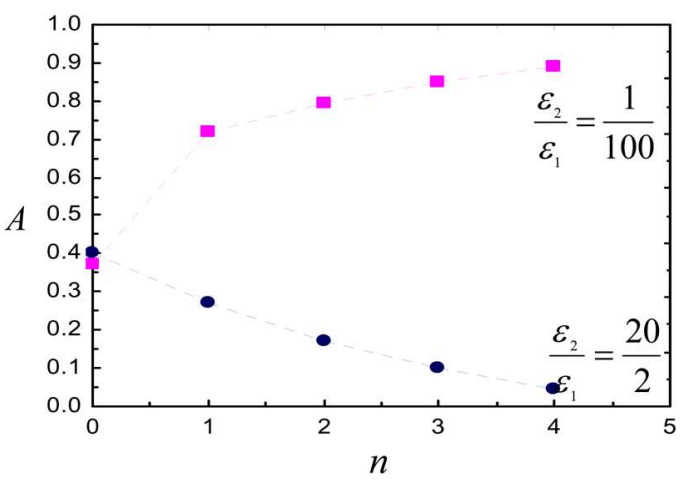

Fig. 6. Iteration number dependence of the DF for the Sierpinski triangle. Full circles correspond to $\left(\varepsilon_{2} / \varepsilon_{1}\right)=(20 / 2)$, and full squares to $\left(\varepsilon_{2} / \varepsilon_{1}\right)=(1 / 100)$. The lines are guides for the eyes.

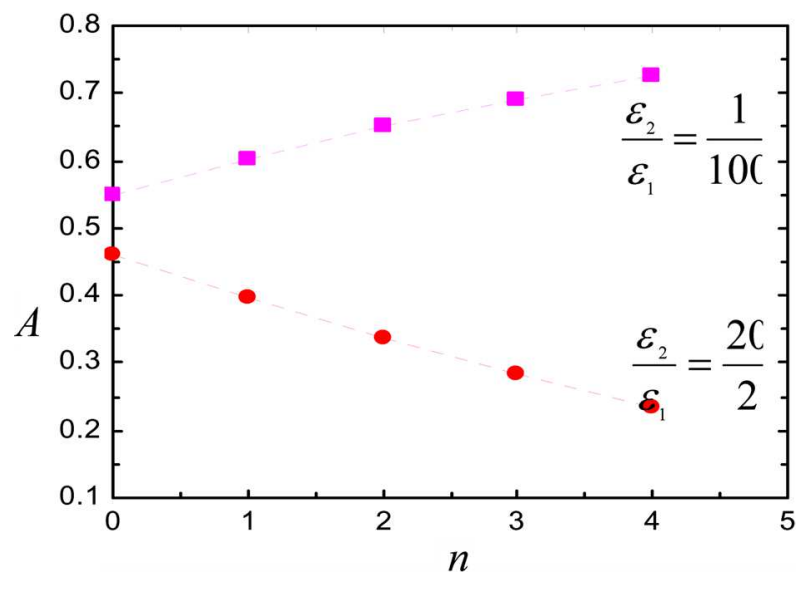

Fig. 7. Same as in Fig. 6 for the Sierpinski square.

we found that the angular dependence of DF is well represented by a $\sin (\theta)$ law.

For comparison and completeness, we have undertaken further calculations on fractal inclusions. The same simulations were run using the same set of parameters for the first four iterations of the fractal patterns. Figs. 6 and 7 suggest that, for sufficiently large iteration number, the DFs converge either to 0 or to 1 .

\section{B. Effect of Varying Permittivity Contrast Ratio}

To further investigate our findings, the same systems were subjected to different permittivity contrast between the con- stituent materials. All other parameters were unaltered. Specifically, we consider a set of situations corresponding to small and large permittivity contrast ratio $\left(\varepsilon_{2} / \varepsilon_{1}\right)$. On examining the data plotted in Fig. 3, we observe that an absolute minimum (respectively maximum), corresponding to the case of disk, for DF is obtained when $\left(\varepsilon_{2} / \varepsilon_{1}\right)<1$ (respectively $\left(\varepsilon_{2} / \varepsilon_{1}\right)>1$ ). Moreover, the values of $A$ are substantially lower (respectively higher) as the number of vertices of the inclusion increases when $\left(\varepsilon_{2} / \varepsilon_{1}\right)<1$ (respectively $\left(\varepsilon_{2} / \varepsilon_{1}\right)>1$ ). this effect can be interpreted as being due to the Keller-Dykhne duality (or phase exchange) relation [2], i.e., $\varepsilon\left(\varepsilon_{1}, \varepsilon_{2}\right) \varepsilon\left(\varepsilon_{2}, \varepsilon_{1}\right)=\varepsilon_{1} \varepsilon_{2}$, which implies that $A \leftrightarrow 1-A$ when $\varepsilon_{1} \leftrightarrow \varepsilon_{2}$. Subsequently, the focus of our attention has been on Sierpinski fractal inclusions. Results in Figs. 6 and 7 suggest two different and opposite trends for this type of inclusion. As before, this behavior originates from the duality symmetry. Moreover, it is found that $A\left(\left(\varepsilon_{2} / \varepsilon_{1}\right) \ll 1\right) \rightarrow 1$ and $A\left(\left(\varepsilon_{2} / \varepsilon_{1}\right) \gg 1\right) \rightarrow 0$ at large iteration number.

For reason of mathematical analogy, the results of this study translate immediately into equivalent results for the demagnetizing factors for a uniformly magnetized inclusion with an arbitrary shape.

\section{Perspectives}

Our approach is expected to be particularly useful to construct models of electromagnetic wave transport in complex 2-D systems, e.g., monolayer colloidal suspensions confined between narrowly spaced glass plates [5], where shape, roughness, and other morphological parameters all contribute to the macroscopic dielectric response. In principle, the method, the FE approach, and our conclusions apply to structures of any dimension.

\section{REFERENCES}

[1] C. Brosseau and A. Beroual, Prog. Mater. Sci., vol. 48, p. 373, 2003.

[2] G. W. Milton, The Theory of Composites. Cambridge: Cambridge Univ. Press, 2002.

[3] A. Mejdoubi and C. Brosseau, Phys. Rev. E, vol. 73, p. 031405, 2006.

[4] E. J. Garboczi and J. F. Douglas, Phys. Rev. E, vol. 53, p. 6169, 1996.

[5] K. Asami, J. Phys. D, vol. 39, p. 492, 2006.

[6] M. L. Mansfield, J. F. Douglas, and E. J. Garboczi, Phys. Rev. E, vol. 64, p. 061401, 2001.

Manuscript received June 24, 2007. Corresponding author: C. Brosseau (e-mail: brosseau@univ-brest.fr). 